\title{
MCATSS - End-to-End Mobile Cardiopulmonary Tolerance Score System
}

\author{
Shravan Aras ${ }^{1}$, Anh Dao ${ }^{2}$, Chris Gniady ${ }^{3}$, Rinku Skaria ${ }^{4}$, and Zain Khalpey ${ }^{5}$ \\ 1,2,3,4,5 University of Arizona, Tucson, 85721, USA \\ shravanaras@cs.arizona.edu \\ adao@cs.arizona.edu \\ gniady@cs.arizona.edu \\ rskaria@surgery.arizona.edu \\ zkhalpey@surgery.arizona.edu
}

\begin{abstract}
Recently hospital re-admission rates have received national attention due to the rise in healthcare costs and poor clinical outcomes specially in case cardiac surgery patients. While there exists risk prediction model for discharging patients, few are utilized due several limitations such as poor discriminative ability, lack of discharge assessment and inadequate measurement of functional status. Further none of the current models present real-time actionable information to facilitate early identification and risk-stratification for patients. Hence in this paper we propose a Mobile Cardiopulmonary Tolerance Score System (MCATSS), which is a system that facilitates data collection using a series of automated tests that incorporate data acquisition from external sensors placed on the patient's body. MCATSS has been designed to reduce erroneous input collection, increase the ease of use for clinicians and provide detailed statistics for medical personals to assist them in determining the safe discharge of patients. Overall, MCATSS offers a platform for digital cross-talk of continuous, sensor- and input-driven data to clinicians at their fingertips and will improve patient care, communication and evidence-based practice while decreasing costs and readmission rates.
\end{abstract}

\section{INTRODUCTION}

Hospital readmission rates have received national attention due to rise in healthcare costs and poor clinical outcomes Desai et al. (2016); Zuckerman et al. (2016). A hospital readmission is when a patient had been released from a hospital and admitted again within 30 days of discharge. Readmission rates have been utilized by hospitals and insurance companies to measure clinical quality and patient care out-

\footnotetext{
Shravan Aras et al. This is an open-access article distributed under the terms of the Creative Commons Attribution 3.0 United States License, which permits unrestricted use, distribution, and reproduction in any medium, provided the original author and source are credited.
}

comes McIlvennan et al. (2015). The establishment of the Hospital Readmissions Reduction Program (HRRP) in 2012 under the Affordable Care Act caused readmission rates to become an important factor in assessing not only the patient care quality but also the healthcare cost. Under the HRRP, hospitals that have high 30-day readmission rates for acute myocardial infraction, heart failure, and pneumonia are financially penalized McIlvennan et al. (2015). In the fiscal year 2013, HRRP penalized 2,200 hospitals \$280 million in Medicare payments, while in fiscal year 2014, 2,225 hospitals were penalized \$227 million in Medicare payments McIlvennan et al. (2015). Since 2014, the HRRP has penalized hospitals for selected clinical conditions including myocardial infarction, heart failure, pneumonia, total knee replacement, and chronic obstructive pulmonary disease Barnett et al. (2015); Desai et al. (2016); Zuckerman et al. (2016). Data from 2017 Centers for Medicare \& Medicaid Services (CMS) reports reveal the mean readmission rates for all-cause, acute myocardial infarction, heart failure, and coronary artery bypass graft procedures were $14.3 \%, 16.3 \%, 21.7 \%$, and $13.8 \%$ respectively.

Although HRRP perhaps has influenced hospital readmission performance, there has been mixed results regarding improvements in clinical outcomes and mortality rates Gupta et al. (2018); Krumholz et al. (2014); Suter et al. (2014), suggesting the need to reassess the HRRP initiative and to improve the procedures and tools for medical personnel to better evaluate patients state and predict which patients are safe to be discharged without risk for readmission with improved accuracy. Existing research in patient's assessment before discharge identify factors and create risk prediction models; however, few are utilized due to many limitations including poor discriminative ability, lack of discharge assessments, and inadequate measurement of functional status. A systematic review by Kangasara et al. presents that only one of the 26 models specifically addressed preventable readmission, seven identified high-risk patients who may benefit from 
intervention early during hospitalization, and five models that could be used at hospital discharge Kansagara et al. (2011).

Variables considered by these studies include sociodemographics, medical diagnoses, illness severity, overall function, utilization metrics, and social determinants of health. Even with the current models used for comparative or clinical purposes, these prediction models perform poorly, and no valid predictive models exist for cardiopulmonary surgery patients. Moreover, no current model presents real-time actionable information to facilitate early identification and risk-stratification of patients. We propose Mobile Cardiopulmonary Ambulatory Tolerance Score System (MCATSS) to incorporate simple noninvasive tests that measure exercise capacity, frailty, pain, and cardiac function and record detailed statistics about each test. The goal of the comprehensive measurements is to determine likelihood of readmission and opportunity to optimize patient health by working to improve their Cardiopulmonary Ambulatory Tolerance Score (CATS) prior to discharge. The proposed system is portable and provides the patient with intervention steps that they can take on their own to improve their CATS prior to surgery or discharge home. In addition, the detailed statistic collected by the MCATSS will enable more comprehensive data analysis to identify features that best correlate to the patient outcomes post-surgery and refine the CATS system. Finally, MCATSS would provide clear and objective tools that allow medical personnel to decide whether patients are safe to be discharged without risk of readmission. Furthermore, these tools will help doctors target patients who most likely benefit from interventions or those who require the most care, which will ultimately reduce hospital readmission.

\section{Motivation}

There are numerous data streams that are cascading into the healthcare field from electronic health records, wearables, metabolomics, and other sources. However, no information is captured and integrated in a useful real-time actionable manner. Current practices to evaluate patient readiness for surgery and discharge involve determining risk factors, monitoring physiologic variables, and undergoing noninvasive tests. However, there are several problems with the current system. Conventionally medical personnel have kept track of information manually, maintaining paper records for their patients. Depending on the case these paper trails can quickly become too complicated to handle. A study conducted by the American Hospital Association found that, for every 30 minutes of care provided to the patient, 30-60 minutes of paperwork was required Association (n.d.). Currently a large number of primary care physicians, around $83 \%-95 \%$ in the United States, still rely on conventional paper records to document and process various clinical data J. Anderson (1999); J. G. Anderson (2007).
Given the lack of standardization to keep track of the various tests performed during pre- and post-operative phases, regarding the number of tests performed or the order in which the tests can be performed. For example, not all clinics do the handgrip test, and even those who do it may not perform it during the appropriate medical phase. Further, given the combination of many tests and already exhausted or tiered personnel, there is a likelihood of some tests being overlooked, incorrect recording of data, or incorrect testing order. A selfreported study conducted by Tang et.al. Tang et al. (2007) of 72 nurses, found that personnel neglect and heavy workload were the leading causes of tests being performed haphazardly. This warrants for a system that can help medical personnel go through the various tests in a predefined order, automatically $\log$ the results as well as notify them or any missed tests.

To address the challenges outlined above we propose MCATSS to allow better data collection, integration, and synthetization of continuous functional data from various tests performed during pre- and post-operative conditions. At present for example, cardiac surgeons rely on traditional risk factor scores including the Society of Thoracic Surgeons (STS) predicted risk. However, they weigh additional frailty tests such as six-minute walk tests, sit-to-stand, and hand grip differently. Some clinicians account these variables in the elderly while others do not. MCATSS offers the opportunity to standardize data capture and improve clinical decision-making process using evidence-based medicine. The collected data can also be used to better assess provider performance by better stratifying patient severity to clinical outcomes. Moreover, it provides incremental prognostic value about a patient's risk for hospital readmission and mortality. Overall, MCATSS offers a platform for digital cross-talk of continuous, sensor- and input-driven data to clinicians at their fingertips and will improve patient care, communication and evidence-based practice while decreasing costs and readmission rates.

\section{Medical Evaluations in MCATSS}

The goals in designing an end-to-end automated MCATSS are: (1) Automate the collection of data using internal / external sensors; (2) Provide automated interventions for tracking patient progress during various peri-operative phases; (3) Reduce errors during patient evaluation steps by medical personnel; and (4) Decrease the effort and time required for medical personnel to record data required by the individual test. Figure 1 presents the implemented MCATSS, consisting of a phone based unified application that is either used by a medical personnel or a patient at home, a NoSql database for storage and retrieval of de-identified (tagged using ids) patient information, and external sensors used to automatically collect physiological data. The MCATTS phone application is unified but presents different functionality based on a login type. Medical personnel are presented with complete testing func- 


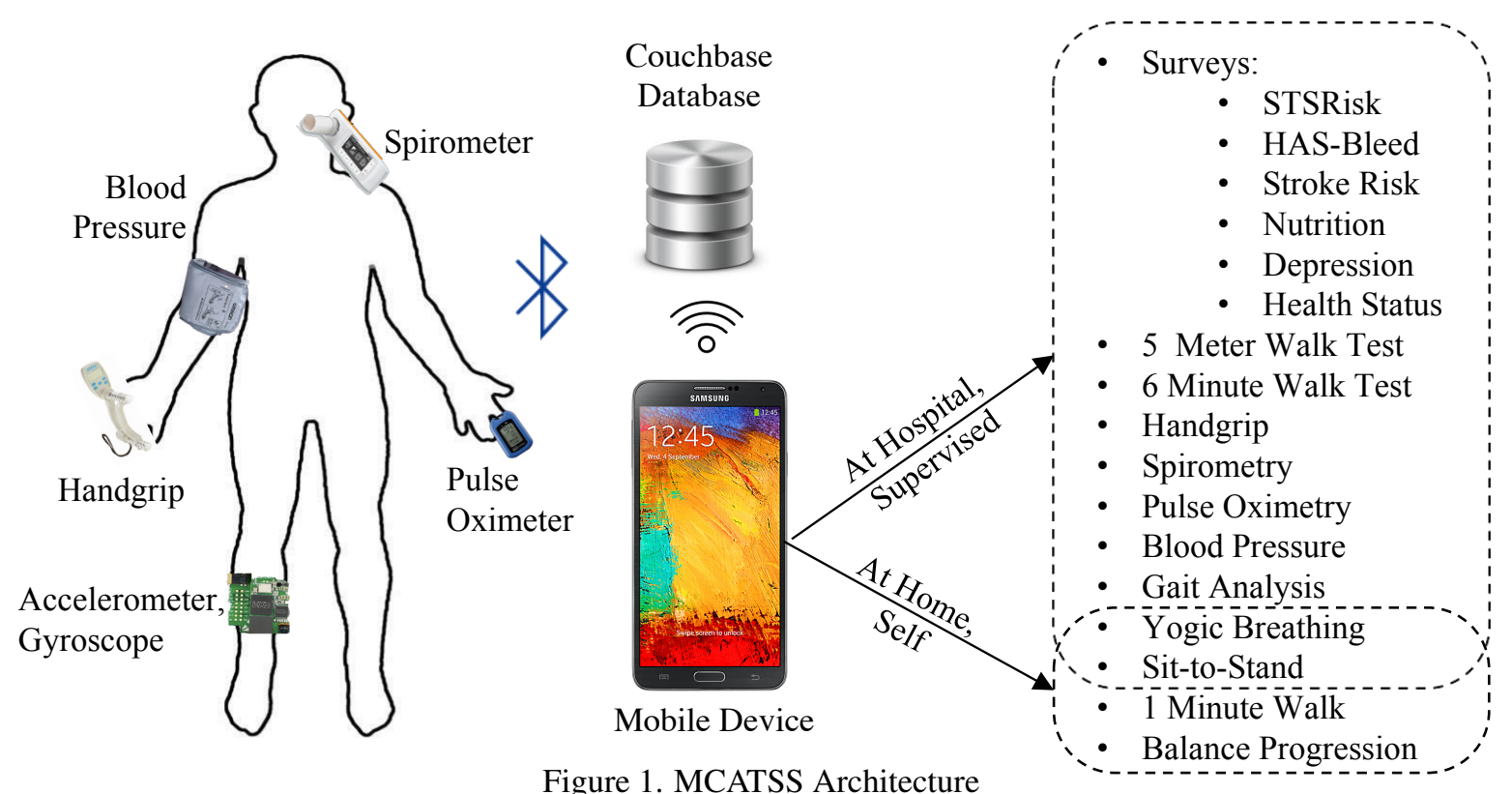

tionality with a guide through various different experiments that they can use to record patient data and several other biomarkers, and patients' information. The patient login results in simplified tests that the patient can perform at home in addition to intervention they can perform to improve their health status. Both intervention and tests for patient offer detailed guidance and verification of completed steps.

Figure 1 outlines in detail the tests and interventions that we implement in MCATSS. The dashed outline separating patient tests and tests supervised by medical personnel can change depending on amount of devices the patient is given to take home. The current outline for medical personnel assumes that all tests that use external sensors are performed at a medical facility; however, if the patient is given some or all devices for home use they will be able to perform the additional evaluation tests at home. The current outline in Figure 1 for at home evaluation test and interventions only requires a phone or a tablet. However, if the patient is prescribed additional tests and given more devices for enhanced monitoring at home, the additional tests will be populated in their MCATSS application and the patient will be guided through them. Some tests like the yogic breathing and sitto-stand are performed in a hospital supervised environment as well as at home by the patients themselves. Depending on the sensors they can be as simple as patient practicing yogic breathing, or they can be combined with vital sign monitoring. The detailed guidance for each test enables even untrained person to follow the directions and perform the measurements.

The external sensors that we use to record patient vitals and statistics are off the shelf components that are inexpensive and readily available. We use an accelerometer and a gyroscope band, which is attached to the person's ankle, for collecting raw data used for automated distance calculation and gait analysis. Pulse oximeter allows us to record oxygen saturation and heart rate in response to various test during the evaluation. Blood pressure monitor allows us to monitor blood pressure at various stages and spirometer collects data on patient lung capacity. Finally, hand dynamometer allows us to measure grip strength for muscle health evaluation.

\subsection{Evaluation Tests in MCATSS}

In order for the medical personnel to comprehensively assess a patients pre-, in-, and post-operative condition MCATSS offers a set of evaluation tests. Each of these tests where selected after careful consultation with the doctors to understand their needs and are carried out in the hospital. Patients wear a pulse oximeter, blood pressure monitor, handgrip, spirometer, and an acceleration band during these tests. Data from these sensors is automatically collected and logged by MCATSS. This sensor integration offers the opportunity to collect and synchronize gait speed and pattern using an accelerometer with heart rate variability, pulse oximetry, etc.; this can help physicians evaluate patient's fall risk, one of the leading causes of hospital readmission and mortality in the elderly. By capturing continuous functional data with heart rate variability in real-time, clinicians can improve clinical decision making using evidence-based medicine. Overall these tests measure the patient's fragility, autonomic functions of the heart and different medical biomarkers and consist of:

- 5-Meter Walk Test - The five-meter walk test is used to predict frailty in patient who are candidates for cardiac surgery. Afilalo et.al. discovered that a patient who takes longer than six seconds to walk five meters at a selfpaced gait speed was at an increased risk for morbidity and mortality after cardiac surgery Afilalo et al. (2010). 
For instance, a patient who takes a longer time to complete the five-meter walk test compared to their baseline will be reminded to complete interventions and tests until their score improves before they are ready for surgery or hospital discharge. For this test MCATSS records how long it takes for a patient to walk 5 meters and their gait using an ankle worn accelerometer band.

- 6-Minute Walk Test - The six-minute walk test assesses gait speed and endurance by measuring the distance a patient can walk on a flat, hard surface in a period of six minutes Wilson et al. (2013). This test has been used to measure functional capacity for patients pre- and postoperation as well as patient's ability for daily activities. It evaluates the global responses of all organ systems involved in exercise including cardiac and pulmonary systems. For this test MCATSS records how long a user can walk in 6 minutes and their gait using ankle mounted acceleration band.

- Sit-To-Stand - The sit-to-stand test is used to quantify the performance of the lower extremity muscles as well as balance after surgery Whitney et al. (2005). For this test the patient is asked to sit down on a chair and then stand up, performing 10 repetitions each. MCATSS records the time and overall posture during the test using ankle worn accelerometer and gyroscope. They are asked to perform a maximum of 10 repetitions. This is another fragility test and this test is used as a practical alternative to manual muscle testing which is not possible to carry out without specialized equipment and trained physicians.

- Yogic Breathing - Yogic breathing takes the user through $4 \mathrm{~s}$ inhale, $4 \mathrm{~s}$ hold, $2 \mathrm{~s}$ inhale, 6s exhale, 2s hold and $2 \mathrm{~s}$ exhale. During this test the patient is also directed to put on the finger-based pulse oximeter using on screen instructions that guide the user through the process. The heart rate data collected during this sequence of controlled breathing is used by doctors to infer the autonomic functions of the heart Raghuraj et al. (1998) as well as an indicator of morbidity. Yogic breathing can also be used by patients at home to improve their cardiopulmonary system output.

- Handgrip - The handgrip assessment is used to measure upper extremity function to determine improvement after cardiac rehabilitation for post-cardiac surgery patients Izawa et al. (2017) in the clinic. Deterioration of skeletal muscle is observed during the postoperative period and is a factor in patient morbidity. This test analyzes physiological outcomes by measuring patient's upper extremity strength by averaging three measurements for both hands. MCATSS uses Bluetooth enabled hand dynamometer handgrips to automatically record statistics during the test.

- Spirometry - Spirometry is a standard clinical test that is used to measure the forced expiatory volume(FEV) and the forced vital capacity(FVC) of pulmonary system in a patient. Doctors use these values to infer odds of pulmonary complications which in turn relates to long-term mortality after surgery Qaseem et al. (2006). MCATSS shows proper usage, guidance, and automatically records statistics from a Bluetooth supported spirometer.

- Blood Pressure \& $\mathbf{S p O}_{2}$ - MCATSS uses Bluetooth enabled blood pressure monitor and pulse oximeters to record standard biomarkers automatically. Blood pressure is an important biomarker to record as high blood pressure is associated with an in-creased risk for cardiovascular events and is one of the three factors for coronary artery disease besides high cholesterol and smoking. Proper Management of blood pressure can reduce patient morbidity and mortality after cardiac surgery.

- Pulse Oximetry $\left(\mathbf{S p O}_{2}\right)$ - MCATSS uses Bluetooth enabled blood pulse oximeter to record heart rate and oxygen saturation during the tests. Those statistics allow clinicians to evaluate cardiac output, need for supplementary oxygen supplies, and overall short and longterm patient outcomes.

\subsection{At Home Assistance and Monitoring by MCATTS}

Besides the hospital evaluations MCATSS application also includes a series of interventions which the patients carry out at home. Intervention and basic evaluation tests only require a phone or a tablet but if desired other devices may be prescribed to the patient to enhance these tests. These interventions are used to improve patients' health status and track their recovery and consist of simple exercises during post-operative stages. MCATSS automatically reminds them about the need to perform interventions, guides them through it, and tracks their activity and results. The interventions where selected by the doctors to meet the need for tracking fragility and the autonomic functions of the heart during the at-home post-operative phase. The MCATSS application implements the following at-home interventions that do not require any additional device beyond a smartphone:

- Walking - The at-home walking test is used to test patients' capacity to perform daily activities after a cardiac surgery. Doctors can use the distance data collected during this test to evaluate the global responses of all organ systems involved thus giving an idea of the patient's fragility. For this test the user is asked to walk for 1 minute around their house while keeping a smartphone in the pocket or waist band. As the patient does this test, the MCATSS application automatically calculates the distance walked by the patients and records it. If prescribed by the physician, the ankle worn accelerometer can provide additional information about gait analysis and more accurate distance measurement.

- Balance Progression - Balance control is a complex action which involves maintaining postures and recovering 
equilibrium. Balance control consists of controlling the body's center of mass over its limit of stability. All these coordinated actions help medical personnel determine the fall risk after a cardiac surgery, another important aspect of fragility which determines the patient's capability to carry out day-to-day tasks without a risk of harming themselves. Physicians also use the results from this test to determine whether a patient needs to undergo balance rehabilitation Mancini \& Horak (2010). This test asks patients to perform 2 tasks - a 1 leg stand for 10 seconds and a full tandem stance for 10 seconds. The MCATSS application has a voice guided countdown timer for both, and collects the accelerometer data about patients' stability during the exercise. The patient is asked to keep the phone in the pocket or waistband, and provide simple feedback after the exercise about difficulty to maintain stability.

- Yogic Breathing - This test was described above for in hospital monitoring. Yogic breathing can be performed as an exercise to improve the health of the patient's cardiopulmonary system. There is no statistic recorded, besides the completion of the exercise. The detailed statistics are only collected if the patient is prescribed pulse oximeter for pulse rate and oxygen saturation monitoring, and in this case the test guides the patient through the tests and measurements.

- Sit-to-stand - This test was also described above for in hospital monitoring. In home setting the patient is told to keep a phone in the pocket or the waistband and the accelerometer statistics are recorded on the phone, indicating the time and ability to perform the exercise. The patent is provided with voice guidance during the test.

\subsection{Surveys in MCATTS}

While quantitative tests which measure specific biomarkers can be widely used for a variety of medical inferences, a patient's medical history and personal lifestyle are difficult to gauge using them. These subjective parameters are used by physicians in conjunction with various quantitative biomarkers to diagnose medical problems as well as tailor treatments specific to individual patients. While the health survey nutrition and depression can be recorded by patients independently, the clinical surveys-STSRIsk, HAS-BLEED and Stroke Risk surveys need to filled out by trained medical personnel. Hence, we implemented the following series of surveys after consultation with the physicians:

- STSRisk - STSRisk survey is a well-known survey by the society of Thoracic surgeons(STS) Shahian \& Edwards (2009). The survey starts off as outlined by STS with list of procedures that the doctor wishes to perform. Doctors complete this survey prior to a cardiac surgery to get a quantified idea about patients' risk of mortality, morbidity, permanent stroke odds, renal failure odds, and reoperation odds. All these metrics are critical for the surgeons to determine the risk of a surgery.

- HAS-BLED - The HAS-Bleed is also a welldocumented survey to determine a score that assesses the 1-year risk of major bleeding in patients with atrial fibrillation. The test was first developed in 2010 using data from 3,978 patients as part of the Euro Heart Survey by Pisters et.al. Pisters et al. (2010). MCATSS uses these published guidelines to calculate a score between 0 and 9 based on eight parameters / questions in the survey, with a score above or equal to 3 indicating a high bleeding risk for atrial fibrillation patients.

- CHADS $_{2}$ Stroke Risk - CHADS 2 Stroke risk stratification score is used by doctors to help determine the 1 year risk of an ischemic stroke in a non-anticoagulated patient with non-valvular atrial fibrillation and has been well documented in past literature as a reliable predictor Olesen et al. (2012). The survey uses a set of 5 parameters / questions to calculate the score and takes into considerations questions related to hypertension, Congestive heart failure (CHF) history and age.

- Nutrition \& Depression - In order for doctors to get a better understanding of patient lifestyle, patients are often asked to fill surveys related to nutrition and depression. This has now become a common practice across several clinics. Nutrition surveys help doctors analyze caloric / protein malnutrition to, for example, help isolate the cause of deficiency such arginase deficiency which can cause the amino acid arginine and ammonia to accumulate gradually in the blood. A condition of ten caused due to high protein meals which can leads to seizures, tremor, and loss of balance. Similarly, the depression survey is a survey outline by Mental Health America America (n.d.), and helps doctors assess the mental state of a patient and possible side effects to certain drugs. Having a digital version of both these surveys in MCATSS reduces the reliance on paper-based surveys which are hard to track and query.

The comprehensive testing, intervention, and surveying of patients provided by MCATSS can significantly simplify and improve data collection, improve standardized scoring systems, and enable building of predictive models to determine patient readiness for surgery and discharge. These models can help personalize interventions and tests for patients based on their scores.

\section{MCATSS IMPLEMENTATION}

MCATSS faces several challenges such as automated data acquisition, secure and robust data storage, easy of use by medical personnel and the patient, and validation of evaluation test data to generate consistent data that can be used for detailed analysis of patient health/fragility status. 


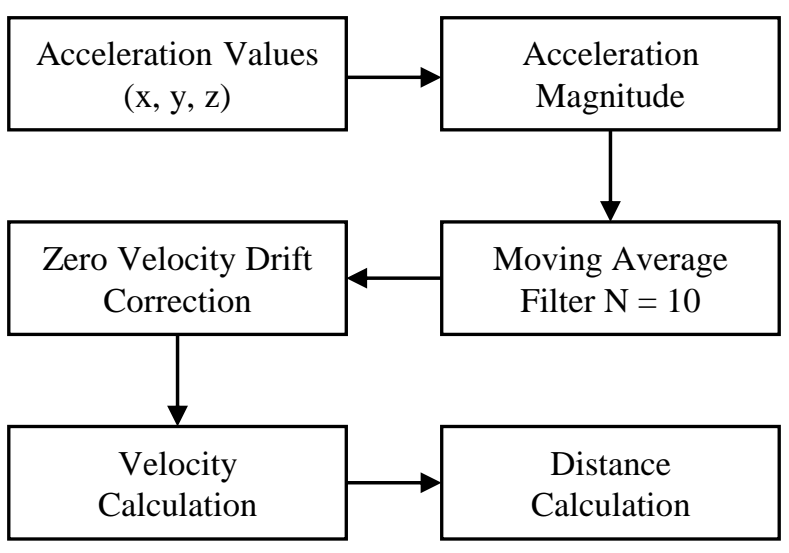

Figure 2. Calculating distance from acceleration data.

\subsection{Automated data acquisition}

MCATSS uses several different sensors to assist in data collection. All of these sensors are connected to the phone using Bluetooth low energy(BLE). Once the phone has established a connection with the peripheral using Generic Access Profile(GAP), we use several different Generic Attribute Profiles(GATT) to query the different characteristics advertised by the various services in order to keep our solution portable. For most of our devices we make use of health profiles defined by the Bluetooth Special Interest Group(SIG); BLP Body blood pressure profile for our blood pressure monitor, PLXP - Pulse Oximeter Profile for our pulse oximeter and Automation IO service for the acceleration band. MCATSS also supports custom GATT profiles, for devices such as spirometers and handgrips, which given the 128 bit characteristic and service UUID are able to read values from the BLE peripheral. This support for custom GATT profiles allows MCATSS to connect to a wide range of nonstandard BLE devices if required.

As one of the goal of MCATSS application is to reduce the effort required in performing various clinical tests, we implemented an automatic distance calculation feature using the acceleration data collected by an accelerator band. We strapped the band onto the ankle using a custom made adjustable strap as shown in the Figure 1. Mounting it on the ankle, as compared to the wrist tends to yield more accurate results due to the stronger leg movement correlation with the motion as compared to wrist movement. When the user selects any movement involving tests, the MCATSS service on the band is automatically started, and starts collecting acceleration and gyroscope data along with the timestamp. The data is stored as shown in Figure 5. In case of the walking tests, the MCATSS automatically calculates the distance walked by the user and either records total distance for the timed walk or how much time the user took to walk a fixed distance.

Figure 2 shows distance calculation steps using acceleration data from the band. These steps are similar to the ones used

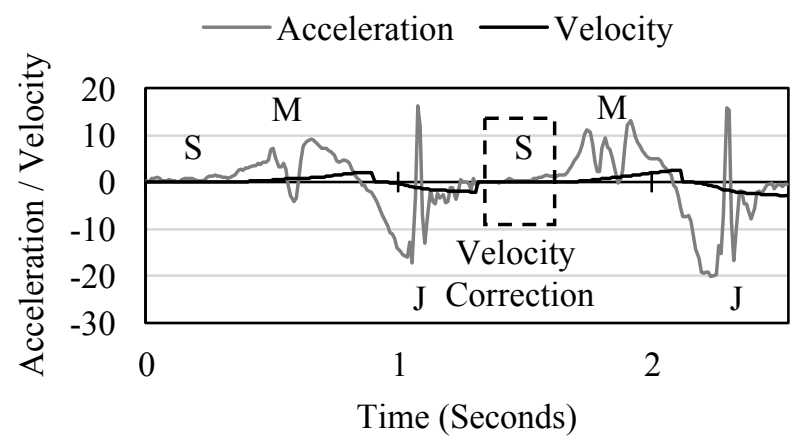

Figure 3. Acceleration and Velocity graph of 2 steps. S : Stationary, M : Start of step, J : Foot hits the ground and step ends.

in past research by Lan \& Shih (2012). We start by calculating the magnitude of acceleration from the raw (x, y, z) acceleration components and smoothen the data to remove any sudden changes caused due to noise, using a moving average filter with a window size of 10 . The data needs to be further filtered as there is a small amount of acceleration captured by the MEMS sensors on the band due to subtle motion of the ankle, even when the foot is on the ground. This adds a drift error to the velocity, resulting in a non-zero velocity value even when the bottom of the foot is stationary. MCATSS applies zero velocity error drift correction, by assuming that when the user's foot touches the ground, the velocity at that point would be 0 since it is in direct contact with the stationary ground.

Figure 3 shows the acceleration magnitude graph when a user takes a single step, labeled with the various components of a step. We see from the figure, that the spike $(\mathrm{J})$ corresponds to the impact of the foot landing on the ground, were as the gradual change in magnitude before that corresponds to the person moving his leg to start the step (M). The stationary portion (S) with the acceleration value close to 0 corresponds the user's foot being in contact with the ground and at rest. MCATSS labels those segments automatically based on the velocity and once the stationary segment is detected, MCATSS zeros velocity in that segment to mitigating the drift error. This can be seen in Figure 3 where the velocity becomes zero when the foot is stationary. Finally, MCATSS converts velocity to speed to calculate the total distance travelled by the user.

The outlined approach was tested on a subject in a confined environment simulating a hospital room or a room in the patient's house. The subject was asked to walk 3 laps, by walking straight line between 2 markers and turning back once they reached the marker. This is common approach to conduct the walking test described in the previous section. The measured total distance using a measuring tape was $25.56 \mathrm{~m}$ in 3 laps. The subject was wearing accelerometer band on the ankle and the magnitudes of acceleration and velocity are shown in Figure 4 Figure 4 also shows the calculated dis- 


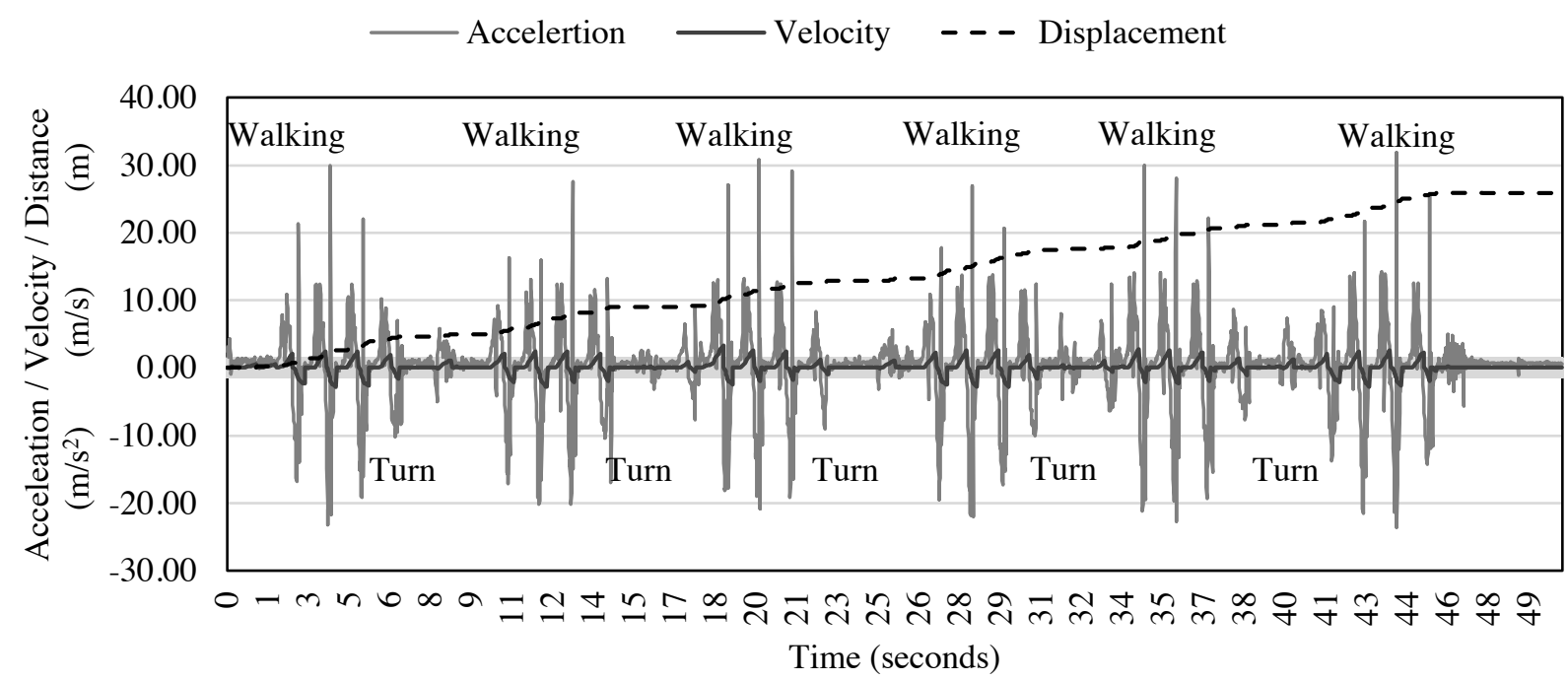

Figure 4. Automatic distance calculation using acceleration data from the ankle mounted accelerometer. (True distance $=$ $25.56 \mathrm{~m}$, Calculated distance $=25.86 \mathrm{~m}$

tance with the zero-velocity drift error correction, walked by the user. We can see from the figure that when the user turns ("Turn" label in the figure), the zero-velocity error correction is employed by MCATSS, preventing the distance value from rising. The MCATSS calculated distance was $25.86 \mathrm{~m}$, resulting in the error rate of only $1.2 \%$. The turns in addition to ankle-based accelerometer placement are critical for employing zero velocity error correction. As seen in Figure 4 the stop $\&$ turn mechanism to walk longer distances in smaller laps gives us $98.8 \%$ accurate distance estimation. This requirement is easy to satisfy as the standard testing procedure is either short walk (5-meter walk test) or longer distance walk but with frequent turns to account for limited distance available in the hospital room.

\subsection{Secure and robust data storage}

The idea behinds MCATSS is to enable comparison of patient parameters during various operative stages - pre, in and post; therefore, we need to index the patient information in a manner that facilitates this co-relation as well as maintains patient anonymity. To anonymize patient data for storage, and enable future analysis without violating patients' privacy, while being able to still look up information for a given patent, we index each patient record using a SHA-256 hash value of 3 parameters. The parameters are entered into the application - First Name, Last Name and Date of Birth. Given a hash's irreversible nature we cannot infer identifiable patient information from the database, thus satisfying the de-identification constrain. However, given the name and date of birth we can recomputed the hash and retrieve corresponding patient's information.

MCATSS uses Couchbase - a distributed NoSQL database that offers access control and encryption to satisfy the 3 re- quirements: (1) Scalability in a distributed manner to keep up with the growing dataset and offer redundancy; (2) Capability of storing heterogeneous data, new data type can be introduced later in the form of additional experiments; and (3) Provide access control and encryption capability. In addition, Couchbase maintains an automatic buffer of unsynchronized data locally (data not yet pushed to the server) and synchronizes it with the remote storage whenever Internet access is available, further simplifying deployment and data collection. Couchbase allows us to create clusters, where we can disrtibute I/O accross different nodes. This increases scalibility by allows MCATSS to distribute reads accross multiple nodes. Data replication on the nodes gives MCATSS avaliablity in case of certain node failures.

\subsection{Evaluation test validation}

Figure 5 shows how MCATSS structures a single session object and stores it into the database. A session is defined by the user logging into the application, selecting the current stage they are in - pre-op, op-day, post-op, discharge, at-home, or others. Each such session contains the timestamp it was started at, the patient ID to which it belongs, a meta-data object and at least one experiment. To increase reliability and decrease the likelihood of data loss, we commit the current state of the session object into our database after completion of each experiment. We call these intermediate commits as sub-sessions. To link these sub-sessions together we use a meta-data object illustrated in Figure 5 The meta-data ID contains a SHA-256 hash of the current timestamp when the session was started. Stage contains one of the many perioperative stages a patient can be in, with optional containing custom stage information, if the selected option was others. This allows MCATSS to quickly link all sessions belonging 


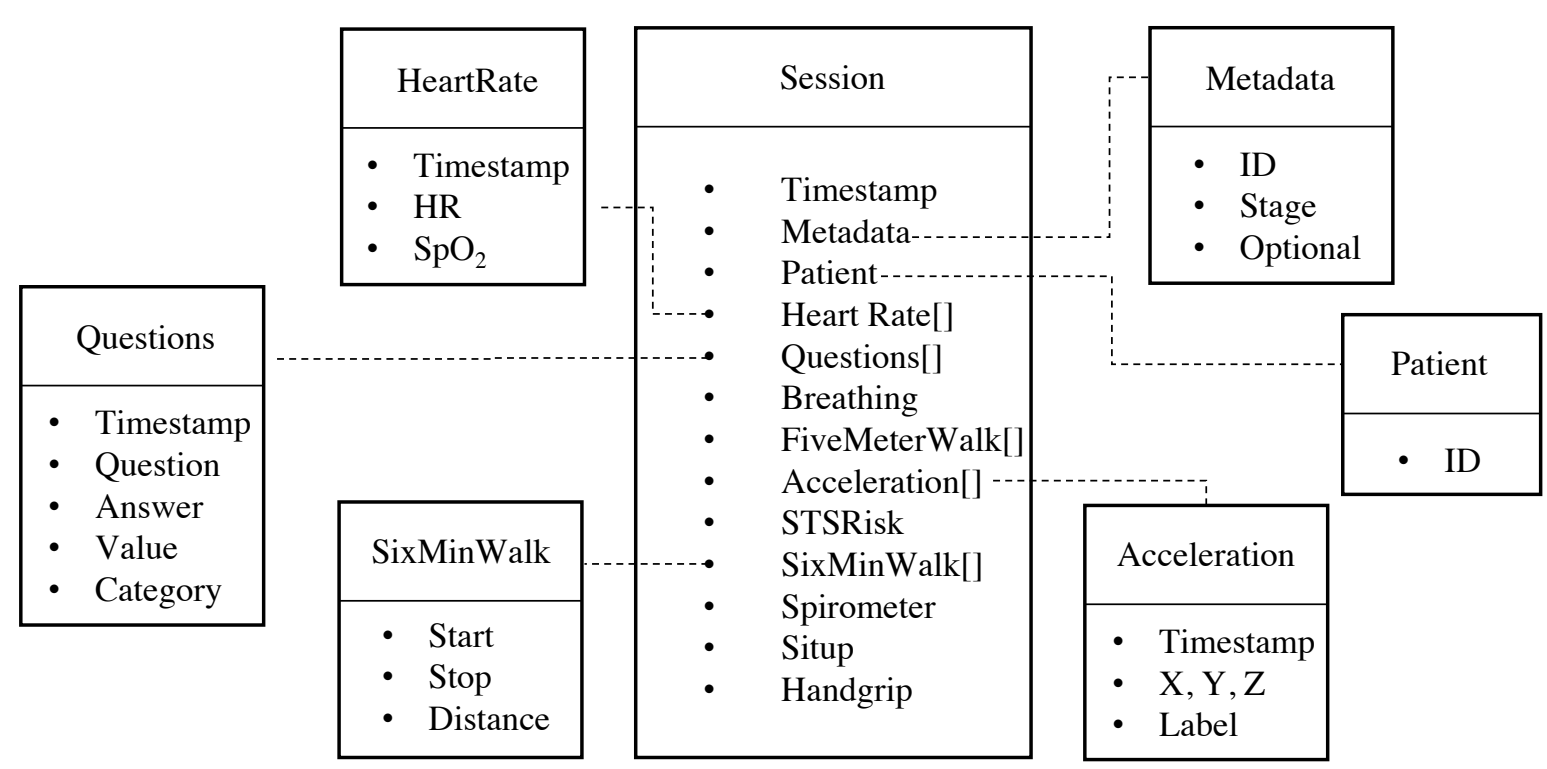

Figure 5. A database representation for each patient evaluation.
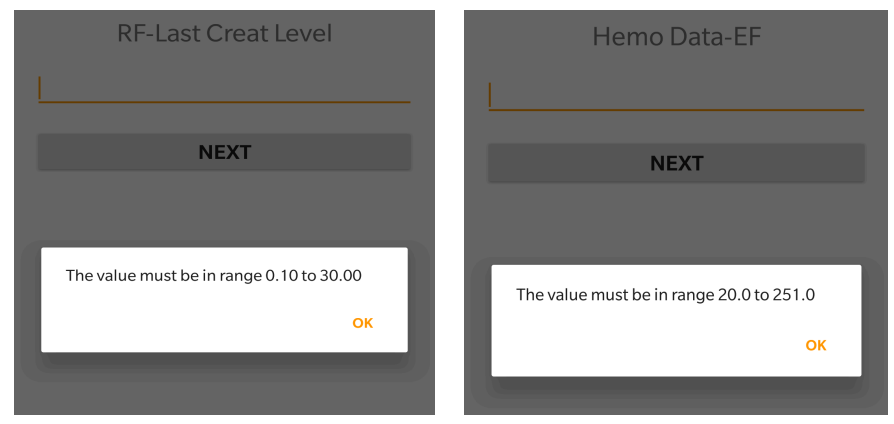

Figure 6. MCATSS performs input validation, checking to see if medical metrics are within the recommended values.

to the same peri-operative stage together to serve queries such as "How did the 6-minute walk test for a particular patient progress during the pre-operative stage and how does it compare to all the walks during the post-operative phase?".

Each session also contains an array of heart-rate and acceleration objects, collected throughout the session using external Bluetooth sensors such as pulse oximeter and ankle worn acceleration bands. MCATSS store the raw (X, Y, Z) values along with their timestamps, accompanied by a label that indicates if the data belongs to the 6-minute walk test or the 5-meter walk test. For each of the two walk experiment objects MCATSS stores the start time, end time and the distance traveled. Since these experiments can be conducted multiple times during a single session, they are stored as an array of objects. Finally, each of the survey questions is also stored as an array of objects as shown in Figure 5. The category indicates to which survey the objects belong to (STSRisk, HASBleed, etc.). The value indicates the numeric score associated with each answer and is used to calculate the overall score a patient gets for the survey.

MCATSS validates the data in several different ways. Due to its use of peripheral devices for automated data collection in case of spirometry, blood pressure, heart rate, oxygen saturation and acceleration values for distance it eliminates errors caused due to manual entry of these biomarkers. After the medical personnel or the at-home patient finishes each test MCATSS automatically synchronizes the data with the Couchbase server. This avoids accidental loss of data which can be caused by nurses or patients forgetting to save the tests or application crashes. Each of the MCATSS test is implemented to have accept a specific set of input and provide a set of outputs. Each of these inputs are validated to make sure the user has entered the correct values. We show 2 such examples of incorrect input errors in Figure 6. In each of those scenarios the user accidentally entered incorrect values for both RF-Last Creat Level and Hemo Data-EF, both of which are parameters used in the calculation of the STSRisk score. Since MCATSS validates all inputs to check if they meet the published guidelines, it rejects the values entered and notifies the user that the values must be within a specific range, 0.10-30 and 20-251 respectively. MCATSS does this for all its input thus further the risk of incorrect data records. All these features, allow MCATSS to accurately record data in both clinical and at-home setting, thus reducing errors caused at source during data collection by neglect or improper usage.

\section{Conclusion}

In this paper we outlined the design and implementation of MCATSS, an end-to-end system that facilitates data collection for the various tests selected for the mobile cardiopul- 
monary tolerance score. We described the various tests selected and implemented such as sit-to-stand, 5-meter walk test, health surveys, various risk surveys (STSRisk, HASBleed); and their clinical significance in patient monitoring. We showed how MCATSS was designed to reduce data acquisition errors by using external Bluetooth sensors to record various biomarkers such as heart rate, blood oxygenation, blood pressure, handgrip, and spirometry values. Further we discussed how MCATSS automates tasks such as distance calculation using ankle worn accelerometer bands, reducing the medical personnel's effort required for fragility tests such as 6-minute walk test and 5-meter walk tests. Besides clinical tests and surveys, MCATSS has also been designed to perform at-home intervention for patients. These interventions have been designed to automatically guide the patient through several tests or exercises they can perform at home using their phone or tablet, thus allowing medical personnel to keep track of their post-operation recovery. Finally, with the widespread adoption and use MCATSS, our system will enable better analysis on the resulting data, giving medical community an in-depth understanding how various biomarkers and tests, during different peri-operative stages, contribute to patient's safe discharge odds.

\section{REFERENCES}

Afilalo, J., Eisenberg, M. J., Morin, J.-F., Bergman, H., Monette, J., Noiseux, N., ... others (2010). Gait speed as an incremental predictor of mortality and major morbidity in elderly patients undergoing cardiac surgery. Journal of the American College of Cardiology, 56(20), 1668-1676.

America, M. H. (n.d.). Depression Survey. http:// screening.mentalhealthamerica.net/ screening-tools/depression. ([Online; accessed 9-Feb-2018])

Anderson, J. (1999). Increasing the acceptance of clinical information systems. MD computing: computers in medical practice, 16(1), 62-65.

Anderson, J. G. (2007). Social, ethical and legal barriers to e-health. International journal of medical informatics, 76(5-6), 480-483.

Association, A. H. (n.d.). Patients or Paperwork. hospitalconnect.com/aha/advocacy - grassroots/advcacy/advocacy/content/ FinalPaperworkReport.pdf. ([Online; accessed 9-Feb-2018])

Barnett, M. L., Hsu, J., \& McWilliams, J. M. (2015). Patient characteristics and differences in hospital readmission rates. JAMA internal medicine, 175(11), 1803-1812.

Desai, N. R., Ross, J. S., Kwon, J. Y., Herrin, J., Dharmarajan, K., Bernheim, S. M., .. . Horwitz, L. I. (2016). Association between hospital penalty status under the hospital readmis- sion reduction program and readmission rates for target and nontarget conditions. Jama, 316(24), 2647-2656.

Gupta, A., Allen, L. A., Bhatt, D. L., Cox, M., DeVore, A. D., Heidenreich, P. A., ... others (2018). Association of the hospital readmissions reduction program implementation with readmission and mortality outcomes in heart failure. JAMA cardiology, 3(1), 44-53.

Izawa, K. P., Kasahara, Y., Hiraki, K., Hirano, Y., \& Watanabe, S. (2017). Relation between the disability of the arm, shoulder and hand score and muscle strength in postcardiac surgery patients. Diseases, 5(4), 31.

Kansagara, D., Englander, H., Salanitro, A., Kagen, D., Theobald, C., Freeman, M., \& Kripalani, S. (2011). Risk prediction models for hospital readmission: a systematic review. Jama, 306(15), 1688-1698.

Krumholz, H. M., Normand, S.-L. T., \& Wang, Y. (2014). Trends in hospitalizations and outcomes for acute cardiovascular disease and stroke: 1999-2011. Circulation, CIRCULATIONAHA-113.

Lan, K.-C., \& Shih, W.-Y. (2012). Using simple harmonic motion to estimate walking distance for waist-mounted pdr. In Wireless communications and networking conference (wcnc), 2012 ieee (pp. 2445-2450).

Mancini, M., \& Horak, F. B. (2010). The relevance of clinical balance assessment tools to differentiate balance deficits. European journal of physical and rehabilitation medicine, 46(2), 239.

McIlvennan, C. K., Eapen, Z. J., \& Allen, L. A. (2015). Hospital readmissions reduction program. Circulation, 131(20), 1796-1803.

Olesen, J. B., Torp-Pedersen, C., Hansen, M. L., \& Lip, G. Y. (2012). The value of the cha2ds2-vasc score for refining stroke risk stratification in patients with atrial fibrillation with a chads 2 score $0-1$ : a nationwide cohort study. Thrombosis and haemostasis, 108(06), 1172-1179.

Pisters, R., Lane, D. A., Nieuwlaat, R., De Vos, C. B., Crijns, H. J., \& Lip, G. Y. (2010). A novel user-friendly score (hasbled) to assess 1-year risk of major bleeding in patients with atrial fibrillation: the euro heart survey. Chest, 138(5), 1093-1100.

Qaseem, A., Snow, V., Fitterman, N., Hornbake, E. R., Lawrence, V. A., Smetana, G. W., ... Owens, D. K. (2006). Risk assessment for and strategies to reduce perioperative pulmonary complications for patients undergoing noncardiothoracic surgery: a guideline from the american college of physicians. Annals of internal medicine, 144(8), 575-580.

Raghuraj, P., Ramakrishnan, A., Nagendra, H., \& Telles, S. (1998). Effect of two selected yogic breathing techniques on heart rate variability. Indian Journal of physiology and pharmacology, 42, 467-472. 
Shahian, D. M., \& Edwards, F. H. (2009). The society of thoracic surgeons 2008 cardiac surgery risk models: introduction. The Annals of thoracic surgery, 88(1), S1.

Suter, L. G., Li, S.-X., Grady, J. N., Lin, Z., Wang, Y., Bhat, K. R., ... others (2014). National patterns of risk-standardized mortality and readmission after hospitalization for acute myocardial infarction, heart failure, and pneumonia: update on publicly reported outcomes measures based on the 2013 release. Journal of general internal medicine, 29(10), 1333-1340.

Tang, F.-I., Sheu, S.-J., Yu, S., Wei, I.-L., \& Chen, C.-H. (2007). Nurses relate the contributing factors involved in medication errors. Journal of clinical nursing, 16(3), 447457.

Whitney, S. L., Wrisley, D. M., Marchetti, G. F., Gee, M. A., Redfern, M. S., \& Furman, J. M. (2005). Clinical measurement of sit-to-stand performance in people with balance disorders: validity of data for the five-times-sit-to-stand test. Physical therapy, 85(10), 1034-1045.

Wilson, C. M., Kostsuca, S. R., \& Boura, J. A. (2013). Utilization of a 5-meter walk test in evaluating self-selected gait speed during preoperative screening of patients scheduled for cardiac surgery. Cardiopulmonary physical therapy journal, 24(3), 36.

Zuckerman, R. B., Sheingold, S. H., Orav, E. J., Ruhter, J., \& Epstein, A. M. (2016). Readmissions, observation, and the hospital readmissions reduction program. New England Journal of Medicine, 374(16), 1543-1551.

\section{BIOGRAPHIES}

Shravan Aras received his Ph.D. in 2018 from Deperatment of Computer Science at the University of Arizona. He received his Bachelors in Computer Engineering from College of Science Pune, India in 2012. His research interests include energy management for mobile and portable healthcare devices - focusing on cellular radios, heart rate monitors in smartwatches and IOT, Operating Systems - energy optimizations for heterogenuous schedulers, Networks and Machine Learning - detection using medical imaging and sequence models. He has won several Gelileo Circle Research awards during his Ph.D. program for his ongoing research efforts.

Anh Dao is a PhD student in the Department of Computer Science at the University of Arizona. He received his Bachelors' degrees in Computer Science and Mathematics from the University of Arizona in 2018. Has been an undergraduate research assistant participating in NSF REU which resulted in paper publication and sever research award from the Uni- versity of Arizona. His research interests focus in the systems area of computer science and mHealth such as designing systems to aid individuals in monitoring personal wellbeing, detect onset of frailty in older adults, and investigating ways to maximize energy efficiency of mobile devices to provide long lasting monitoring.

Chris Gniady is an Associate Professor in the Department of Computer Science at the University of Arizona. He received Ph.D. in 2005 and B.S. in 1997 in Electrical and Computer Engineering from Purdue University. His research interests include energy management from mobile phones to datacenter and supporting networking infrastructure, performance optimizations at hardware and software levels, and novel applications of sensor and mobile devices in healthcare. He is a member of IEEE, ACM, and USENIX and has published papers in prestigious venues such as USENIX OSDI, ACM SIGMETRICS, IEEE HPCA, ACM ISCA, and IEEE TOCs. He has served on technical program committees of many conferences and workshops. He is also a recipient of NSF CAREER award for research in energy optimization based on user interactions.

Rinku Skaria is an MD/PhD student at the University of Arizona College of Medicine. She received her Bachelors' degrees in Physiology and Business Management from the University of Arizona in 2014. She has been involved with the American Medical Student Association since 2010. As president of the association, she worked alongside a team offering clinical, philanthropic and social events for the UA PreHealth Professions Expo Week. She also worked with middle school students from educationally disadvantaged populations, students with learning disabilities, and promoted higher education and team values. Her research interests include cardiovascular disease with focus on preventing and mitigating postoperative surgical outcomes.

Zain Khalpey is a cardiothoracic surgeon at Northwest Healthcare in Tucson. He completed medical school at Guy's, Kings and St Thomas Hospital, London School of Medicine and continued his training in the U.S., with a residency and fellowship at Brigham and Women's Hospital, Harvard Medical School in Boston. This was followed by a superfellowship in mechanical circulatory support devices for advanced heart failure at Columbia University's New York Presbyterian Hospital. He completed a PhD in Cardiothoracic Surgery, Bioenergetics and Cardiac Transplantation through the Imperial College UK in London with work conducted at the University of Gdansk in Gdansk, Poland, the Mayo Clinic in Rochester, MN, and Harvard University in Boston. In 2016, he was awarded the Distinguished Fulbright Chair in Medical Sciences, one of the most prestigious appointments in the Fulbright Scholar Program. He has a special interest in sudden cardiac death, robotic mitral valve repair, total artificial hearts, and using short- and long-term devices in shock and heart failure patients. 